\title{
Atrial tachycardia, atrioventricular conduction disturbance and right heart failure years after removal of a cardiac hydatid cyst
}

\author{
Christos - Konstantinos Antoniou ${ }^{1}$, Petros Arsenos ${ }^{1}$, Nikos Apostolopoulos ${ }^{1}$, Polychronis Dilaveris ${ }^{1}$, Skevos Sideris ${ }^{2}$, Dimitris Tsiachris ${ }^{1}$, \\ Ioannis Skiadas ${ }^{2}$, Elias Sotiropoulos ${ }^{2}$ and Konstantinos A Gatzoulis ${ }^{1 *}$ \\ ${ }^{1}$ Electrophysiology Unit, First Division of Cardiology, Athens Medical School, Hippokration General Hospital, 114 Vasilissis Sofias ave. 11527, Athens, Greece \\ ${ }^{2}$ State Cardiology Division, Hippokration General Hospital, 114 Vasilissis Sofias ave. 11527, Athens, Greece
}

\begin{abstract}
The present case report article focuses on the two-decade-long follow-up of a patient presenting with a rare echinococcosis complication, the formation of a hydatid cyst in the interventricular septum. Following successful surgical removal she experienced numerous complications, including atrial tachycardia, severe tricuspid regurgitation with right heart failure and aborted sudden death. Consequently, she was submitted to a host of invasive procedures, namely tachycardia ablation, valvular surgery (twice) and cardioverter - defibrillator implantation. This case is interesting because, despite the fact she experienced numerous complications, both from her disease and its treatment (conduction disturbances, valvular insufficiency leading to surgery and aborted sudden cardiac death) she is currently well and largely asymptomatic.
\end{abstract}

\section{Patient history}

A 28-year old woman was evaluated in 1995 due to precordial discomfort associated with protracted fever and leukocytosis. Echocardiography revealed an echolucent cystic mass in the interventricular septum (IVS) and MRI scans showed a hypointense mass on T1 sequence, occupying most of the IVS (Figure 1). Following positive serology assays, cardiac hydatid disease was diagnosed. Patient was referred for surgery and following resection antiechinococcal treatment was administered. No other cysts were discovered following extensive imaging workup.

Six years later, she presented complaining of paroxysmal palpitations, dyspnea and fatigue over the last five months. An electrocardiogram showed atrial tachycardia (AT) at 130 beats per minute with 2:1 atrioventricular conduction and right bundle branch block with left anterior hemiblock (Figure 2a). P wave morphology was positive in aVL, isoelectric in I and negative in V1. Electrophysiology study revealed a markedly prolonged $\mathrm{H}-\mathrm{V}$ interval of 170 milliseconds and unstable atrioventricular conduction deteriorating to complete block upon fast pacing (Figure 2b).

An ablation catheter located the point of the earliest atrial activation at the inferior mid-anterior right atrial wall (Figure 3a) where radiofrequency ablation terminated the arrhythmia (Figure 3b). A dual chamber pacemaker was implanted to ensure proper atrioventricular conduction. Patient recovered uneventfully; however, paroxysmal AT relapsed and, being asymptomatic, declined further ablation attempts.

Over the next years, 24-hour electrocardiogram recordings consistently showed AT paroxysms and variable atrioventricular conduction, with normal pacemaker function.

In 2003, on follow-up, she complained of exertional dyspnea (NYHA II) and a parasternal systolic murmur was audible. Echocardiography demonstrated severe tricuspid regurgitation with dilation of the right cardiac chambers; thus, after initial medical management tricuspid valve repair with annulus support was performed in 2005.

An episode of aborted sudden cardiac death led to upgrading to a single-chamber (VVI) defibrillator (2009) while relapse of the severe tricuspid regurgitation led to repeat tricuspid repair two years later. Patient has since experienced two ICD activations (one shock and one antitachycardia pacing for sustained ventricular tachycardia episodes both at $>190 \mathrm{bpm}$ ), however has otherwise been asymptomatic (pacemaker dependent, significant improvement in right ventricular function and size).

\section{Discussion}

Echinococcosis is an important parasitic zoonotic disease, with an annual incidence of 0.4 per $10^{5}$ [1]. Humans are accidental hosts and usually infected by ingestion of eggs by drinking contaminated water or handling egg-containing soil, dirt or animal hair. Ingested eggs release embryos that can localize to any organ and grow into hydatid cysts, evading immunological detection [2] and reaching large volumes. Symptoms depend upon location and size of the cyst with rupture leading to anaphylaxis [3].

Correspondence to: Konstantinos A. Gatzoulis, Electrophysiology Unit, First Division of Cardiology, Athens Medical School, Hippokration General Hospital, 114 Vasilissis Sofias ave. 11527, Athens, Greece, Tel: (0030)6944580369; E-mail: kgatzoul@med.uoa.gr

Key words: cardiac echinococcosis, atrial tachycardia ablation, atrioventricular block

Received: September 03, 2017; Accepted: September 25, 2017; Published: September 27, 2017 


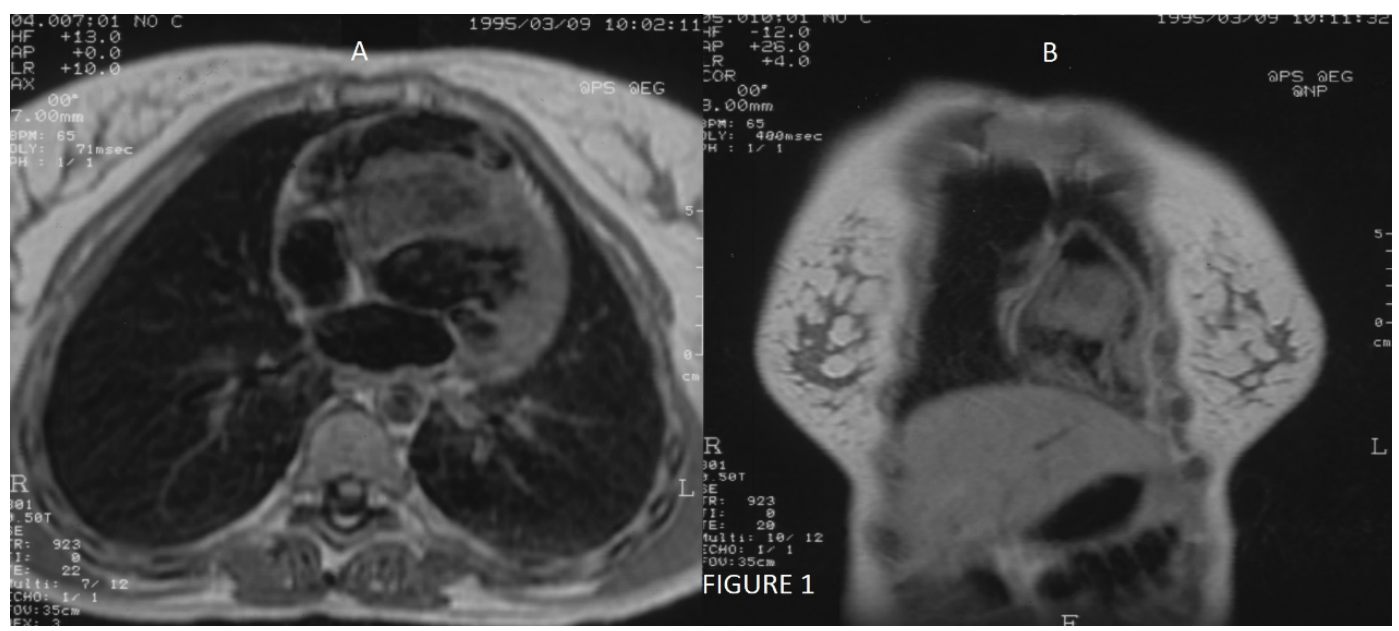

Figure 1. MRI scan of the lesion, T1 sequence, transverse (A) and frontal (B) plane. A hypointense cystic mass ( $4.8 * 2 \mathrm{~cm}$ on the transverse plane and $2.4 * 3.7 \mathrm{~cm}$ on the frontal plane) is occupying the IVS, almost obliterating the right ventricle.
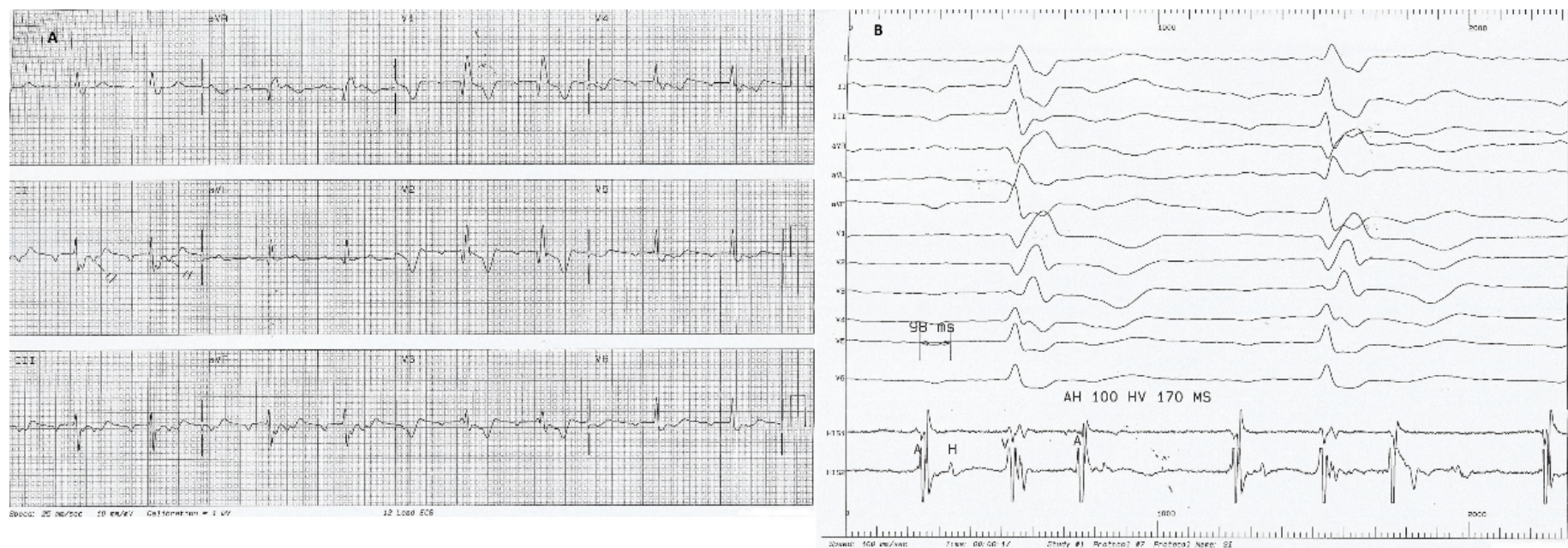

Figure 2.

A.12-lead ECG of atrial tachycardia. Note inverted P waves in inferior leads, positive in leads aVR, aVL and isoelectric in lead I with negative-positive polarity in lead V1. 2:1 atrioventricular block with prolonged PR interval, along with right bundle branch block and left anterior hemiblock.

B.Electrogram showing marked prolongation of the His-ventricle interval with 2:1 conduction at the level of the AV node. Surface ECG leads followed by His bundle electrograms during tachycardia.

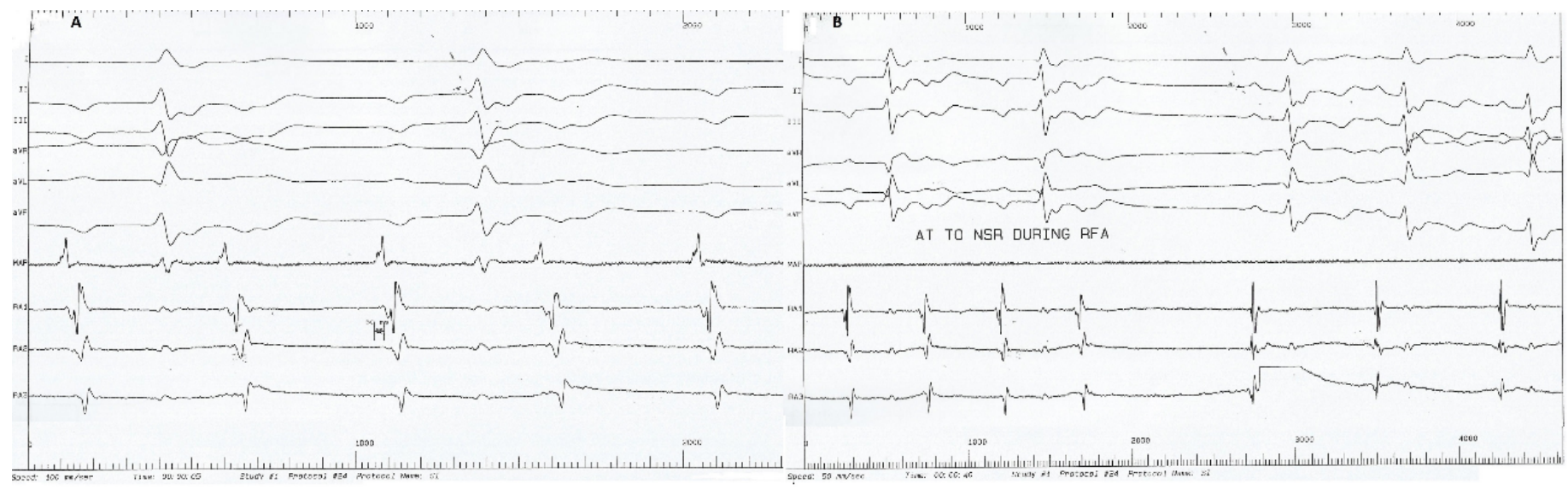

Figure 3.

A. Localization of atrial tachycardia focus. When the ablation catheter was placed in the inferior mid-anterior right atrial wall, its electrogram preceded that of the $\mathrm{P}$ wave by $30 \mathrm{msec}$.

$\mathrm{B}$. Ablation of arrhythmogenic focus and conversion to sinus rhythm with 1:1 atrioventricular conduction and first degree block. Surface leads followed by catheter recordings (MAP) and 3 adjacent poles of an atrial electrode catheter (RA 1-3). 
The heart is affected in $0.5-2 \%$ of cases [3]. Cardiac involvement, mainly of the left ventricle (60\% of cases), usually occurs through either the coronary blood flow or rupture of a lung hydatid cyst into a pulmonary vein [4]. Localization to the interventricular septum is even rarer ( $4 \%$ of cardiac echinococcosis [5]). Intracavitary cyst expansion is ominous due to high rupture risk. Diagnosis is made by ultrasonography and treatment of choice is surgical removal with gentle handling to avoid rupture, followed by antihelminthic medication. MRI is the modality of choice to determine the exact anatomic location and borders while CT best depicts calcification [6].

IVS surgery carries the risk of complications regarding both the valvular and conduction structures nearby [7]. Thus, it is conceivable that our patient's tricuspid regurgitation and atrioventricular block emerged as post-operative complications, the latter after fibrosis scar developed. Furthermore, fibrosis could have generated the substrate for AT paroxysms, a connection supported by focus location (lower part of the right atrium).

$\mathrm{AT}$ is the least frequent form of supraventricular arrhythmia with an atrial rate in the 150-200 beats per minute range and can be caused by re-entry, abnormal automaticity, or triggered activity. It usually presents in patients with significant structural heart disease but may also occur in structurally normal hearts, or with digitalis intoxication, as either paroxysmal or incessant $(25 \%)$ arrhythmia. It is a long RP - short PR tachycardia. Although relatively benign, it may cause, when incessant, tachycardia-induced cardiomyopathy. Symptoms usually depend on underlying pathology and most notable findings are the supernumerary jugular $\alpha$ waves and the variability in the S1 loudness and blood pressure levels, due to the alterations in the PR interval and varying atrioventricular block. Thus, in this patient with a compromised conduction system, superimposition of AT predictably led to atrioventricular block.

Although initial treatment is medical (beta and calcium channel blockers, with class I or III antiarrhythmics added if necessary), treatment of choice in cases of symptom recurrence, incessant tachycardia or tachycardia-mediated cardiomyopathy is catheter ablation [8]. Recurrence can occur from a different focus or over a different re-entry circuit.
Tricuspid valve repair was performed twice on the patient due to recurrent severe regurgitation, heart failure symptoms affecting quality of life and causing enlargement of the right side of the heart. Given her young age, repair was considered preferable to replacement, with the latter to be performed at an older age, if necessary. Conceptually, it is reasonable to attribute the aborted sudden death episode and the two ICD activation incidents to right ventricular failure-related arrhythmogenesis.

This case of cardiac hydatid cyst location is interesting insofar as the young patient experienced numerous complications from removal surgery (atrioventricular block leading to pacemaker implantation, atrial tachycardia and tricuspid regurgitation), had a non-lasting arrhythmia ablation and two cardiac valve surgeries, experienced right heart failure, as well as an aborted sudden cardiac death event leading to defibrillator upgrade, providing further life-saving therapy twice, and yet, today, at age 48 , she is alive and well, with a good overall functional status.

\section{References}

1. Abhishek V, Avinash V (2012) Cardiac hydatid disease: literature review. Asian Cardiovasc Thorac Ann 20: 747-750.

2. Siracusano A, Delunardo F, Teggi A, Ortona E (2012) Host-parasite relationship in cystic echinococcosis: an evolving story. Clin Dev Immunol 2012: 639362. [Crossref]

3. Mandal S, Mandal MD (2012) Human cystic echinococcosis: epidemiologic, zoonotic, clinical, diagnostic and therapeutic aspects. Asian Pac J Trop Med 5: 253-260. [Crossref]

4. Younis SN, Faraj AA (2014) Cardiac hydatid disease, case report, and review of literature. Acta Clin Belg 69: 66-68. [Crossref]

5. Shankarappa RK, Moorthy N, Bhat P, Nanjappa MC (2013) Left ventricular hydatid cyst: an uncommon cause of chest pain in young adults. Cardiol Young 23: 460-462. [Crossref]

6. Dursun M, Terzibasioglu E, Yilmaz R, Cekrezi B, Olgar S, et al. (2008) Cardiac hydatid disease: CT and MRI findings. AJR Am J Roentgenol 190: 226-232. [Crossref]

7. Butchart EG, Gohlke-Bärwolf C, Antunes MJ, Tornos P, De Caterina R, et al. (2005) Recommendations for the management of patients after heart valve surgery. Eur Heart $J$ 26: 2463-2471. [Crossref]

8. Lee G, Sanders P, Kalman JM (2012) Catheter ablation of atrial arrhythmias: state of the art. Lancet 380: 1509-1519. [Crossref]

Copyright: (C2017 Antoniou CK. This is an open-access article distributed under the terms of the Creative Commons Attribution License, which permits unrestricted use, distribution, and reproduction in any medium, provided the original author and source are credited. 